\title{
Agreement between indirect measures of perceived distance
}

\author{
WALTER C. GOGEL, JACK M. LOOMIS, NANCY J. NEWMAN, and THOMAS J. SHARKEY \\ University of California, Santa Barbara, California
}

\begin{abstract}
A test configuration consisting of either one or two points of light was made to appear at different distances on different trials. The perceived distance of the configuration on each trial was measured indirectly using two procedures. In the size adjustment procedure, the observer adjusted the vertical separation of two equidistant points to produce a criterion-perceived separation of $1 \mathrm{in.}$ On the basis of this adjustment, the perceived distance of the two-point configuration was derived by means of the size-distance invariance hypothesis (Gilinsky, 1951; Kilpatrick \& Ittelson, 1953). In the head-motion procedure, the observer indicated the motion of a physically stationary test point as it appeared to move concomitantly with the head. On the basis of the direction and magnitude of this apparent motion, the perceived distance of the test point was derived by means of the apparent-distance/pivot-distance hypothesis (Gogel, 1982). The measures obtained from these two procedures correlated highly. Since the size adjustment procedure provides a measure of perceived distance that generally is accepted as valid, the results indicate that the head-motion procedure has a similar validity. Past research with the head-motion procedure often concerned the apparent motion or apparent distance of a single object presented in a dark surround. An analysis of the present results supports the conclusion that the apparentdistance/pivot-distance hypothesis applies equally to situations in which objects in addition to the test object are present.
\end{abstract}

There are two general methods for measuring perceived distance. One is to obtain from the observer a direct response to distance, for example, a verbal report of distance in feet and inches. The other is to measure perceived distance indirectly by making use of the relation between perceived distance and some other percept. For instance, the perceived distance of an object can be computed from the size-distance invariance hypothesis (SDIH) (Gilinsky, 1951; Kilpatrick \& Ittelson, 1953) by using the visual angle of the object together with a measure of its perceived size as obtained from the observer. In applying such indirect measures of perceived distance, it is improbable that the observer is aware of the law or rule relating the several percepts. It is unlikely, therefore, that the observer will attempt to modify the response to the related percept (perceived size in the case of the SDIH) in an effort to achieve a more accurate measure of perceived distance as calculated from the perceptual interrelation. On the other hand, indirect measures must meet a requirement not found with direct measures. This requirement is that the relation between the percepts must be known to the experimenter before the measure of perceived distance can be calculated.

An indirect measure of perceived distance obtained using a method called the head-motion procedure has been found to be useful in evaluating the effectiveness of differ-

This study was supported by a United States Public Health Service Grant MH 39457 from the National Institute of Mental Health. The authors' mailing address is: Department of Psychology, University of California, Santa Barbara, CA 93106. ent cues of perceived distance (Gogel, 1976, 1981; Gogel \& Tietz, 1976). It has also been useful in evaluating the effect of suggestion upon verbal reports of distance (Gogel, 1981; MacCracken, Gogel, \& Blum, 1980), in concluding that errors in perceived distance occur frequently, particularly as a function of the distance of fixation (Gogel, 1979), and in providing principles that can be applied to three-dimensional illusions of motion (Gogel, 1980). The primary objective of the present study was to further examine the head-motion procedure by comparing measures of perceived distance obtained using this procedure with measures obtained from applying the SDIH.

The equation for the SDIH used in the present study was that

$$
D^{\prime}=S^{\prime} D / C S
$$

where $S^{\prime}$ is perceived size, $D^{\prime}$ is perceived distance, $S$ and $\mathrm{D}$ are physical size and physical distance, respectively, and $\mathrm{C}$ is an observer constant. For the purposes of the present analysis, it will be assumed that $\mathrm{C}=1$. The head-motion procedure uses a direct observer response to an apparent motion of a test object associated with a lateral motion of the head. Applying the apparentdistance/pivot-distance hypothesis, the experimenter relates this response to perceived distance. The apparentdistance/pivot-distance hypothesis is that

$$
W^{\prime}=K\left(1-D^{\prime} / D_{p}\right)
$$


or, solving for $\mathrm{D}^{\prime}$, that

$$
\mathrm{D}^{\prime}=\mathrm{D}_{\mathrm{p}}\left(\mathrm{K}-\mathrm{W}^{\prime}\right) / \mathrm{K} .
$$

In Equations 2 and 3, $W^{\prime}$ is the perceived extent of motion of the test object, $\mathrm{K}$ is the lateral extent of motion of the head, $D^{\prime}$ is the perceived distance of the test object, and $D_{p}$ is the distance of a hypothetical (or actual) point (the pivot distance) around which the direction between the head and test object pivots as the head is moved laterally (Gogel, 1982). ${ }^{1}$ According to Equation 2, for a given magnitude, $\mathrm{K}$, of head motion, the only factor determining the perceived extent of motion of the test object is the ratio $D^{\prime} / D_{p}$. Conversely, from $W^{\prime}$ and $D_{p}$, perceived distance, $\mathrm{D}^{\prime}$, can be calculated using Equation 3 .

The way in which the size-distance invariance hypothesis is used in the study is discussed in relation to Figure 1. The apparent-distance/pivot-distance hypothesis and its application to the study are considered in relation to Figure 2. Definitions of the physical and perceptual terms used in the figures and throughout the text are listed in the appendix.

Figure 1 illustrates the situation in which the physical vertical separation $\left(S_{n}\right.$ or $S_{f}$ ) of two test points (indicated by the solid circles in $A$ and $B$ ) is varied by the observer until their perceived separation achieves a constant criterion, in the present study an apparent separation of $1 \mathrm{in}$. In the situation illustrated, the physical distance (D) of the test points from the observer is constant, but the perceived distance, $D^{\prime}$, of the points, indicated by the open circles, is greater in $A$ than in $B$ (i.e., $D_{f}^{\prime}>D_{n}^{\prime}$ ). In order for the perceived vertical separation of the two test points to be the same in $A$ and $B$, that is, in order that $S_{\mathrm{f}}^{\prime}=S_{n}^{\prime}$,
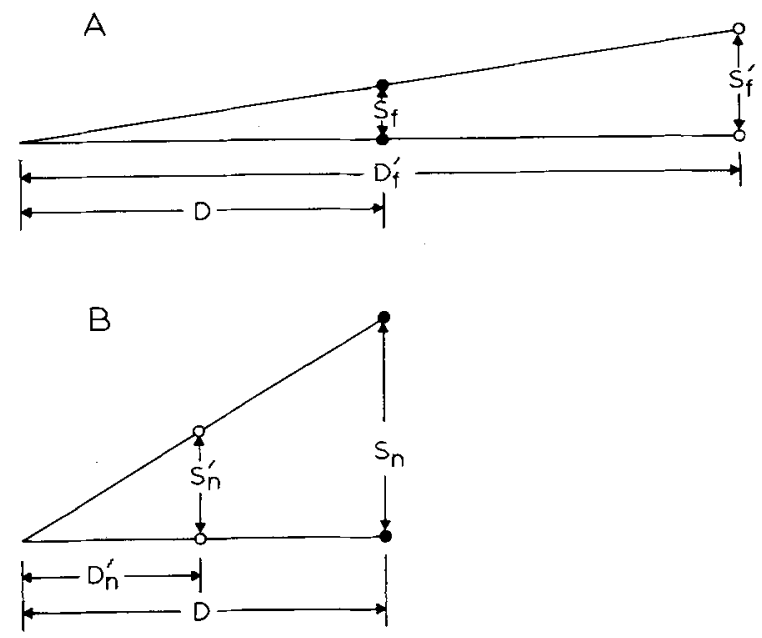

Figure 1. Applications of the size-distance invariance hypothesis (SDIH) in which the physical vertical separation $\left(S_{n}\right.$ or $\left.S_{i}\right)$ of two points (filled circles) at a constant physical distance (D) is adjusted to appear (open circles) equal to 1 in. The primed and unprimed notation indicates physical and apparent quantities, respectively. The perceived distance of the points is greater in $A$ than in $B$, and bence, according to the SDIH, the physical separation required to produce a perception of $1 \mathrm{in}$. should be greater in $B$ than in $A$. it follows from the SDIH that the physical, adjusted separation $S_{n}$ must be greater than the physical, adjusted separation $S_{f}$. Conversely, from the physical separation, $S_{n}$ or $S_{f}$, required to achieve the constant perceived separation of 1 in., the perceived distance, $D_{f}^{\prime}$ or $D_{n}^{\prime}$, of the test points can be calculated using Equation 1. This value, calculated from Equation 1, is called the perceived distance from the size-adjustment procedure and is labeled $D_{s}^{\prime}$.

The application of the apparent-distance/pivot-distance hypothesis to the present study is illustrated in Figure 2. The solid circles in Figure 2 represent three possible physical distances $\left(D_{n}, D_{m}\right.$, or $\left.D_{f}\right)$ of a single test point, and the open circles represent three possible perceived distances of the test point from the observer $\left(D_{\mathrm{n}}^{\prime}, D_{\mathrm{m}}^{\prime}\right.$, or $\left.\mathrm{D}_{f}^{\prime}\right)$ for each of the physical distances as the head is moved laterally through a physical distance $K$. In the situations shown, and in the present study, the test point always is physically stationary. The solid circles in this case also represent the physical distance positions $\left(D_{p}\right)$ around which the direction from the observer to the test point pivots as the head is moved laterally. For a stationary test point, $\mathrm{D}_{\mathrm{p}}$ always is identical to the physical distance of the test point. ${ }^{2}$ The basic assumptions of Figure 2 are that the pivot angle $\left(\phi_{\mathrm{p}}\right)$ and the amount of head motion $(\mathrm{K})$ are perceived correctly. If these assumptions are met, as seems to be the case (Gogel, 1982), the test object will appear to move $\left(\mathrm{W}^{\prime}\right)$ concomitantly with the motion of the head whenever $D^{\prime} \neq D_{p}$. The perceived distance $D_{n}^{\prime}$, $D_{m}^{\prime}$, or $D_{f}^{\prime}$ of the test point for a given $D_{p}$ and $K$ can be calculated by the experimenter from $\mathrm{W}^{\prime}$ by applying Equation 3. This calculated value of perceived distance obtained using $\mathrm{W}^{\prime}$ and the apparent-distance/pivot-distance hypothesis as expressed by Equation 2 is called the perceived distance from the head-motion procedure and is labeled $D_{w^{\prime}}^{\prime}$. As shown in Figure 2, $W^{\prime}$ will be in the same direction as the head motion (a plus $\mathrm{W}^{\prime}$ ) whenever $D^{\prime}<D_{p}$ and opposite to the direction of the head motion (a minus $\mathrm{W}^{\prime}$ ) whenever $\mathrm{D}^{\prime}>\mathrm{D}_{\mathrm{p}}$. Taking the sign of $W^{\prime}$ into consideration, it is evident from Figure 2 that for a constant $D_{p}, W^{\prime}$ will decrease with increasing $D^{\prime}$, and for a constant $\mathrm{D}^{\prime}, \mathrm{W}^{\prime}$ will increase with increasing $D_{p}$ or, since the test point is physically stationary, with increasing physical distance (D) of the test point.

A second objective of the study is to examine whether the apparent-distance/pivot-distance hypothesis, which has been shown to apply successfully to a test object presented in a dark surround (Gogel, 1982; Gogel \& Tietz, 1979), will apply also when objects in addition to the test object are visible. For this purpose, the visual alley in which the test object was presented was lined with a patterned (polka dot) cloth. Throughout this study, the observation of the test point and the visual alley was binocular. Thus, binocular disparities between the test point and the polka dots were available to perceptually localize the test point in apparent depth. Depth cues of relative accommodation between the test point and polka dots also were present. In addition, as the head was moved laterally, a gradient of relative motion parallax was produced between the test 


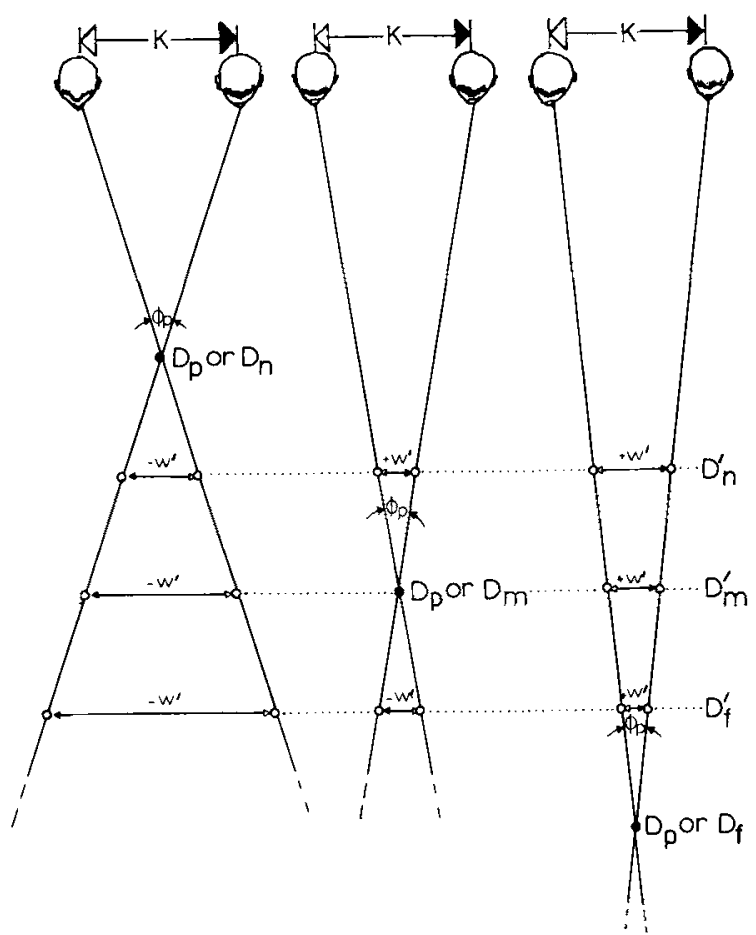

Figure 2. The apparent motion $\left(W^{\prime}\right)$ of a physically stationary test point viewed with a moving head as a function of its pivot distance $D_{p}$ and apparent distance $D_{n}^{\prime}, D_{m}^{\prime}$, or $D_{f}^{\prime}$. The primed and unprimed notation indicates physical and apparent quantities, respectively, with $\phi_{\mathrm{p}}$, the angle formed at the pivot distance as measured from the extremes of head motion, $K$.

point and polka dots. These cues, by contributing to the $\mathrm{D}^{\prime}$ of the test point for a particular $\mathrm{D}$ of the test point, also would be expected to contribute to the adjusted $S$ in the size-adjustment procedure and to the measured $\mathrm{W}^{\prime}$ in the head-motion procedure. The motions on the eye of the images of the polka dots relative to the image of the test point, as a consequence of the lateral motion of the head, warrant special consideration. Suppose that such relative retinal motions, independent of any effect they might have on the $\mathrm{D}^{\prime}$ of the test point as a consequence of relative motion parallax, significantly modified the $\mathrm{W}^{\prime}$ of the test point in a manner similar to an induced motion (Duncker, 1929). In this case, the calculated $D^{\prime}$ from the head-motion procedure $\left(D_{w^{\prime}}^{\prime}\right)$, since it is based on $W^{\prime}$, would be distorted. The $\mathrm{D}^{\prime}$, as measured by the sizeadjustment procedure $\left(D_{s}^{\prime}\right)$, however, since it is based upon the adjusted $S$ and not on $W^{\prime}$, would not be modified by an induced motion. As a result, $D_{w^{\prime}}^{\prime}$ and $D_{s}^{\prime}$ would not exhibit strict covariation. The question is whether the apparent-distance/pivot-distance hypothesis can apply to situations in which the test object is presented in the context of other objects, as well as presented alone. If this hypothesis is to apply, with $\mathbf{K}$ held constant, $\mathrm{W}^{\prime}$ must be determined exclusively by the ratio of perceived distance to pivot distance and not by factors such as an induced motion, which, if effective, could modify ' W' without modifying this ratio. To test this possibility, dif- ferent gradients of relative retinal motion were produced by varying the pivot distance of the test point. Varying the pivot distance of the test point in this study was achieved by varying its physical distance. With the observer's gaze maintained on the test point throughout the motion of the head, the physical distance of the display monitor (the pivot distance) changed the proportion of polka dot images moving across the retina in a given direction (i.e., in the same direction as, or in a direction opposite to, the head motion).

Three pivot distances $\left(D_{p}\right)$ were used for each of three perceived distances ( $\left.D_{I}^{\prime}\right)$. The pivot distance of the test point was changed by changing the physical distance of the display monitor on which the test point was produced. The perceived distance of the test point was changed by instructing the observer to adjust its convergence distance until the test point appeared at a specified distance $\left(D_{1}^{\prime}\right)$. The pivot distance $\left(D_{p}\right)$ of the test point was independent of both its convergence distance $\left(D_{\alpha}\right)$ and the instructed perceived distance ( $\left.D_{1}^{\prime}\right)$. This is illustrated in Figure 3. For simplicity, the instructed perceived distance $\left(\mathrm{D}_{\mathrm{I}}^{\prime}\right)$ is drawn as equal to the convergence distance $\left(D_{\alpha}\right)$, although, as the results will show, $D_{I}^{\prime}$ and $D_{\alpha}$ often were not the same. Polarizing filters at the appropriate orientations imaged a different point of light from the monitor on each eye. The physical separation of these two points on the monitor together with the orientation of the polarizing filters (and the observer's interpupillary distance) specified the convergence distance $\left(\mathrm{D}_{\alpha}\right)$ of the binocularly fused, single point of light. In this study, $D_{p}$ was always
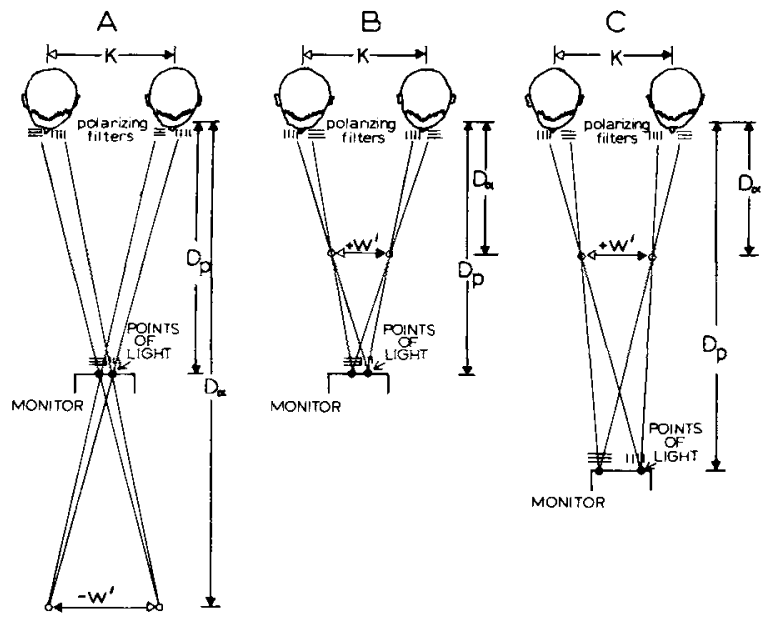

Figure 3. Diagrams illustrating the independence of the convergence distance $\left(D_{\alpha}\right)$ and the pivot distance $\left(D_{p}\right)$. In this study, the stimulus points on the display monitor remain physically stationary as the head is moved. In this case, the physical distance of the display monitor (D) is always the same as the pivot distance $\left(D_{p}\right)$. The convergence distance, on the other hand, is determined by the separation of the points on the display monitor, the interpupillary distance of the observer, and whether the polarizing filters (shown by the sets of small parallel lines) provide a convergence behind (A) or in front of (B or $C$ ) the monitor. The value of $D_{\alpha}$ is adjusted by the observer until the test point appears at 3,5 , or $7 \mathrm{ft}$. The value of $D_{p}$ is set by the experimenter to be at 135,185 , or $235 \mathrm{~cm}$. 
the same as the physical distance of the monitor. The independence of $D_{p}$ from $D_{\alpha}$ (and $D_{1}^{\prime}$ ) is emphasized in Figure 3 in that for the same $D_{p}$, the adjusted $D_{\alpha}$ (and $\left.D_{I}^{\prime}\right)$ differ between $A$ and $B$, whereas for the same $D_{\alpha}$ (and $\left.D_{I}^{\prime}\right), D_{p}$ differs between $B$ and $C$. Also, it should be noted that in $A, D_{\alpha}$ is behind the monitor, whereas in $B$ and $C$ it is in front of the monitor. The change in $D_{\alpha}$ between $A$ and $B$ in this case is accomplished as shown, by switching the orientation of the polarizing filters in front of the two eyes of the observer.

To produce the three pivot distances $\left(D_{p}\right)$, the monitor was placed at 135,185 , or $235 \mathrm{~cm}$ from the observer. To achieve the three apparent distances $\left(D_{\mathrm{I}}^{\prime}\right)$, the observer adjusted the convergence distance $\left(D_{\alpha}\right)$ by changing the separation of the points on the monitor and, if necessary, the orientations of the polarizing filters until the test point appeared at a distance $\left(\mathrm{D}_{\mathrm{I}}^{\prime}\right)$ of 3,5 , or $7 \mathrm{ft}(91.4,152.4$ or $213.4 \mathrm{~cm}$ ) as instructed by the experimenter. The details of the method by which the adjustment of $D_{\alpha}$ was accomplished will be described shortly. For each of the nine combinations of $D_{p}$ and $D_{I}^{\prime}$ (three values of $D_{p}$ for each of the three values of $\left.D_{1}^{\prime}\right)$, the physical size (S) needed to achieve a constant perceived size $\left(\mathbf{S}^{\prime}\right)$ and the measured apparent motion $\left(\mathrm{W}^{\prime}\right)$ concomitant with the motion of the head were used in the above equations to calculate $D_{s}^{\prime}$ and $D_{w^{\prime}}^{\prime}$. These measures then were compared to determine the agreement between the apparent distance calculated from the SDIH and from the apparent-distance/pivotdistance hypothesis.

\section{METHOD}

\section{Observers}

The observers were four men and two women who were paid for participating in the experiment. Two of the observers were undergraduate and four were graduate students in the Psychology Department. Each had an uncorrected visual acuity of at least $20 / 20$ in each eye and a stereoscopic acuity of $11.0^{\prime \prime}$ of arc or better. Two observers, who were unable consistently to either binocularly fuse the test point of light or adjust the convergence to achieve the instructed apparent distances $\left(D_{\mathrm{I}}^{\prime}\right)$, were replaced in the study.

\footnotetext{
Apparatus

Experimental situations. The visual alley and associated apparatus used in the experimental situations are illustrated schematically in Figure 4. The visual alley was $31 \mathrm{~cm}$ wide, $93 \mathrm{~cm}$ high, and $245 \mathrm{~cm}$ long. Polka dot (white and black) cloth covered the floor and side walls of this alley. The white dots were $2.7 \mathrm{~cm}$ in diameter with $5.2 \mathrm{~cm}$ between centers. The ceiling of the alley was covered by uniform black cloth, and black cloth formed the back wall. The alley was illuminated throughout its length by a fluorescent light mounted on the ceiling. A light-tight booth at the front of the alley contained the observation position, with the front of the observation booth forming the front wall of the alley. A viewing aperture, $33 \mathrm{~cm}$ long $\times 11 \mathrm{~cm}$ high, in this wall permitted the observer to see into the alley when a shutter controlled by the experimenter was raised. The eyes of the observer were approximately $26 \mathrm{~cm}$ above the alley floor. Two partially reflecting, partially transmitting first surface mirrors allowed the observer, when the shutter was open, to see simultaneously the visual alley and a small (2$\mathrm{mm}$ ) point of white light at eye level, apparently suspended in this alley but actually generated in an adjacent and parallel nonvisible alley, as illustrated in Figure 4. This point-of-light (the test object),
}

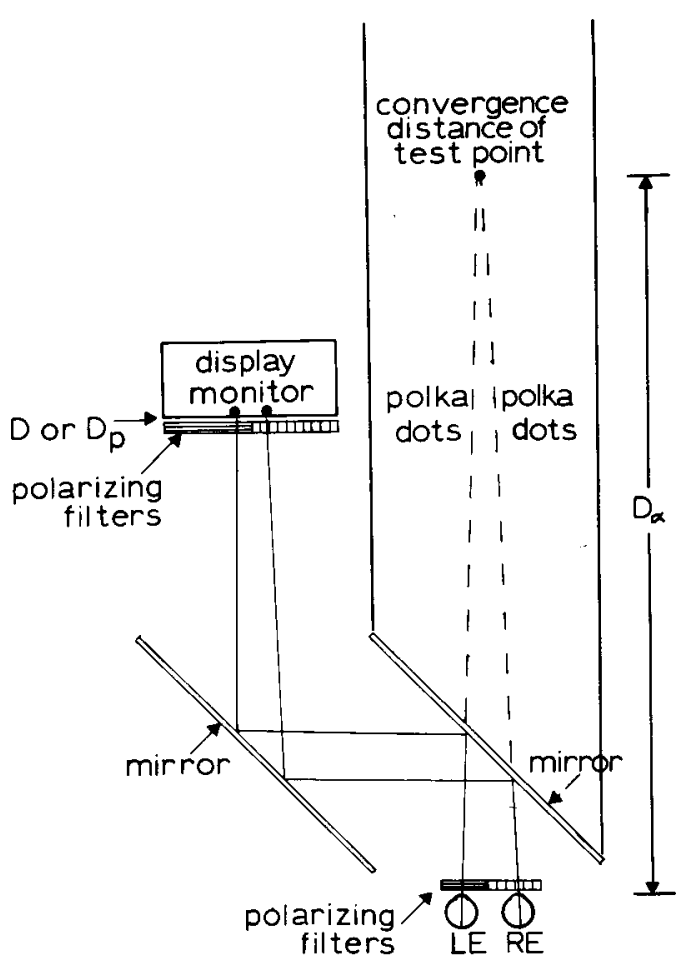

Figure 4. Method of producing a luminous test point in a visual alley lined with a polka dot cloth. The test point generated in the adjacent alley on a display monitor is variable in convergence disance $D_{\alpha}$. To generate the test point, two points of light on the display monitor, capable of being varied in their horizontal separation, are reflected by two mirrors into the eyes of the observer. One point is seen by the left eye only, and the other point by the right eye only (as determined by pairs of Polaroid filters located at the surface of the monitor and at the position of the observer). Reversing the Polaroid filters at the position of the observer reverses the convergence distance of the test point from a distance behind to a distance in front of the optical distance to the display monitor. The pivot distance $\left(D_{p}\right)$ of the test point always is at the physical distance, $D$, of the monitor. This distance is the distance from the cyclopean eye of the observer as measured along the lines that include the reflections from the mirrors. In this study, $D_{p}$ and $D$ were always identical.

as described previously with respect to Figure 3, was, in fact, two points of light generated by a computer on the display monitor in the adjacent alley. As controlled by the computer, the observer could simultaneously move the points horizontally in opposite directions on the display monitor, to provide the variable horizontal separation required for the convergence adjustments. One pair of polarizing filters was located at the surface of the monitor. The other pair was mounted in a frame attached to a chinrest at the observation position and located directly in front of the observer. The polarizing filters permitted one of the two points of light to be seen only by the right eye and the other only by the left eye of the observer. By turning a knob located near the observation position, the observer varied the convergence distance to the single, binocularly fused "simulated" point of light in the visual alley. Changing the convergence of the test point changed the binocular disparity between the test point and the polka dots distributed along the visual alley. This resulted in the observer's being able to move the test point in apparent depth continuously from a near to a far distance. By reversing the polarizing filters at the observation position, as instructed by the experimenter, the observer changed the conver- 
gence for a given horizontal separation of the points on the monitor surface from a convergence distance behind the monitor to a convergence distance in front of the monitor or vice versa. A label at the top of the filters allowed the observer to communicate the orientation of the polarizing filters to the experimenter. To position it at each of the three $D_{p}$ (or $D$ ) values, the monitor could be moved in distance along a track parallel to the floor of the visual alley. The convergence distance adjusted by the observer was computed, taking into account the interpupillary separation of the observer's eyes, the horizontal separation of the points on the monitor surface, the arrangement of the polarizing filters, and the distance $\mathrm{D}$ of the monitor from the observer. The observer could vary the convergence distance $\mathrm{D}_{\alpha}$ of the test point from well behind to well in front of the distance of the monitor for each of the three monitor distances. As computed for the observers' average measured interpupillary distance of $6.31 \mathrm{~cm}$, the range of convergence distances extended from beyond optical infinity to a near distance of 39.2, 53.8 , and $68.3 \mathrm{~cm}$ for the $D_{p}$ of 135,185 , and $235 \mathrm{~cm}$, respectively. With the observation booth light turned off and the visual alley illuminated, nothing was visible to the observer except the alley, the polka dots, and the single, binocularly fused point of light perceptually located in this visual alley. The average brightness of the point of light at the three physical distances following $20 \mathrm{~min}$ of dark adaptation was $2.3 \mathrm{log}$ units above threshold.

The chinrest could be pinned by the experimenter to be directionally at the center of the visual alley, or it could be freed to be moved by the observer on ball bearings from the right to the left of this center through a total lateral distance of $21.5 \mathrm{~cm}$, as specified by stops at the right and left extent of motion. When the observer was told to begin moving his head in the chinrest from one stop to the other, a metronome producing a click every $1.5 \mathrm{sec}$ was turned on to pace the arrival of the chinrest at the stops. Below the track on which the chinrest moved, two vertical posts were located, each with a square cross section of $1.6 \mathrm{~cm}$ on a side and a height of $8.3 \mathrm{~cm}$. The right post was fixed in position, and the left post was moveable laterally. A small black curtain covered this portion of the apparatus and, using touch alone, the observer adjusted the lateral separation of the inner surfaces of the posts to equal the magnitude of the lateral motion that was perceived in the point of light when the head was moved laterally between the stops. A microphone and speaker system enabled the metronome to be heard by the observer and permitted the experimenter and observer to communicate.

Both experimental and calibration situations were used in the study. The experimental situations consisted of the nine combinations of the three $D_{I}^{\prime}$ and three $D_{p}$ values. In the experimental situations, the observer had three tasks. One was to adjust the convergence of the point of light until, as instructed by the experimenter, it appeared to be 3,5 , or $7 \mathrm{ft}$ from himself. Upon achieving this adjustment, the convergence to the test point $D_{\alpha}$ and the physical or pivot distance of the test point $\left(D_{p}\right)$ were unchanged for the remaining tasks to be completed for that combination of $D_{p}$ and $D_{\alpha}$. Another experimental task was to report verbally the apparent direction of the lateral motion of the test point relative to the direction of the head motion, and, by adjusting the movable post, to indicate tactually the magnitude of the lateral apparent motion of the test point, if indeed the test point was perceived to move. This apparent motion $\left(W^{\prime}\right)$, whenever it occurred, was entirely illusory, since the test point always was physically stationary. Another experimental task was to adjust the physical separation (S) of two vertically separated points of light until this separation appeared to equal 1 in. For this task, a second pair of points of light, one presented to each eye by means of the polarizing filters, was produced on the monitor surface, and located directly above the first pair. The binocularly fused upper and lower points were always at the same convergence distance, $D_{\alpha}$, previously adjusted by the observer for the lower point in response to the $D_{I}^{\prime}$ instruction. The upper point could be moved by the observer directly up or down relative to the fixed lower point by means of two buttons located at the observation position. Pressing one button increased and pressing the other decreased the physical vertical separation of the these points.
Calibration situations. In addition to the nine experimental situations, two calibration situations were used. It is probable that observers differ as to the physical size they require to produce an appearance of $1 \mathrm{in}$. or the physical distance they require to produce an apparent distance of 3,5 , or $7 \mathrm{ft}$. The purpose of the calibration situations was to evaluate the possible effect of such differences on the study. ${ }^{3}$ In one calibration situation, the accuracy of indicating the physical size of 1 in. was tested. From a distance of about $38 \mathrm{~cm}$, the observer binocularly viewed a second monitor placed in a lighted area. The observer adjusted the vertical distance between two points of light on the monitor by pressing keys on a computer until the vertical separation of the points appeared to be 1 in. Five adjustments, each preceded by a random vertical separation of the points set by the experimenter, were obtained and recorded from each observer without the observer's being given any information as to the accuracy of the adjustments. The distance calibration was obtained in a different alley, which was illuminated throughout its length and located in a separate room. The alley was $95 \mathrm{~cm}$ wide and $359 \mathrm{~cm}$ long, with checkerboard cloth covering the floor and white cloth on the walls. By pressing a switch in one or the other direction, a metal rod, $1.1 \mathrm{~cm}$ in diameter and extended vertically $29 \mathrm{~cm}$ above the alley floor, could be made to move along the length of the alley. The rod protruded from a narrow slit in the center of the alley floor. The motor, the chain, the cart, and the track for moving the rod were hidden below the alley floor. The observer, using binocular observation, adjusted the rod to appear at 3,5 , or $7 \mathrm{ft}$, with the same random sequence of these adjustments being used twice. The assumption of the calibration situations is that, since much visual information was available, size and distance were correctly perceived and errors in the adjustments reflected differences in the subjective standard of what constituted a particular size or distance.

\section{Procedure}

Prior to entering the observation booth, the observers were given preliminary instructions regarding their tasks; during the experiment, they were given specific instructions. Each observer was presented with the nine experimental and two calibration situations. The different experimental situations were presented to the observer twice in the same order, with a different order of $D_{1}^{\prime}$ and of $D_{p}$ being given to each observer. One presentation of the experimental situations occurred in the first session and the second occurred in the second session, with the two sessions separated by approximately 1 week and each lasting about $1.5 \mathrm{~h}$. The calibration situations always were presented following the two experimental sessions. For half the observers, the size calibration preceded the distance calibration; for the other half, the order was reversed.

In the experimental situations, the task of adjusting the test point of light in convergence distance $\left(\mathrm{D}_{\alpha}\right)$ to appear at the instructed distance ( $\left.D_{1}^{\prime}\right)$ was always completed before a measure of the direction and magnitude of the apparent motion $\left(\mathrm{W}^{\prime}\right)$ concomitant with the motion of the head was obtained and before the size adjustment (S) to equal an apparent extent of 1 in. was made. The $D_{\alpha}$ adjusted to achieve a particular $D_{1}^{\prime}$ always was maintained for the immediately following $\mathrm{W}^{\prime}$ and $\mathrm{S}$ tasks. The latter two tasks were completed in one order for half the observers and in the reverse order for the other half. The three tasks ( $D_{I}^{\prime}, W^{\prime}$, and S) were completed for a particular pivot (physical) distance $D_{p}$ of the test point before the test point was presented at a different $D_{p}$.

The $D_{1}^{\prime}$ task was accomplished as follows: With the chinrest pinned in place, the observer adjusted the convergence to the point until it appeared approximately at 3,5, or $7 \mathrm{ft}$ as instructed. Following this, the chinrest was unpinned by the experimenter from a position outside the observation booth, the metronome was turned on, and the observer moved his head from right to left through four cycles while completing the adjustment to the instructed apparent distance. The purpose of making the first part of the adjustment with the head in a fixed position was to avoid a large number of head motions during the convergence adjustment, which could lead 
to some adaptation of the perceived motion (Hay, 1971; Tietz \& Gogel, 1978; Wallach \& Kravitz, 1965; Wallach, Yablick, \& Smith, 1972). Upon completion of the convergence adjustment, the control of convergence was removed from the observer so that an accidental modification of the control could not occur while the following $\mathrm{S}$ and $\mathrm{W}^{\prime}$ tasks were being completed.

The size-adjustment task was accomplished in a similar manner. For the first, approximate, adjustment of the vertical separation of the centers of the two points to an apparent separation of 1 in., the chinrest was fixed in place, but in the latter part of the adjustment, it was moved laterally for four cycles.

The W' task had two parts. In the first part, the observer moved his head laterally for four cycles and then indicated verbally whether the point appeared stationary or appeared to move in the same direction as ("with") or opposite to ("against") the head. If illusory concomitant motion was reported, four additional cycles were completed in which the observer noted the magnitude of the apparent motion. The shutter then was closed and the observer adjusted the lateral separation of the two measurement posts by touch to indicate the magnitude of the perceived lateral motion of the point.

\section{RESULTS}

\section{Experimental Situations}

A summary of the results from the experimental situations for the $\mathrm{D}_{\alpha}, \mathrm{W}^{\prime}$, and $\mathrm{S}$ tasks are shown in Table 1 . The data are the results obtained from the six observers for the nine $D_{1}^{\prime}, D_{p}$ combinations averaged over both the observers and the two sessions. The entries in the columns and rows labeled "means" are the averages of the data in the indicated columns or rows with one of the variables, $D_{1}^{\prime}$ or $D_{p}$, constant. Consider the effect of the observer's adjustment of the convergence of the point of light to achieve the apparent distance ( $\left.D_{1}^{\prime}\right)$ of 3,5 , or $7 \mathrm{ft}$ upon (1) the resulting convergence distance $\left(D_{\alpha}\right),(2)$ the apparent motion $\left(\mathrm{W}^{\prime}\right)$ concomitant with the motion of the head, and (3) the adjusted vertical size (S) needed to achieve a perceived frontoparallel size $\left(S^{\prime}\right)$ of 1 in. It is likely that the most important determiner of the perceived distance of the test object in the study was the binocular disparities between the test object and the polka dots distributed along the alley. The perceived distance from this factor, of course, is expected to increase with increases in the convergence distance to the test object. Consistent with this expectation, as shown in Table 1 , to achieve a greater $D_{I}^{\prime}$, the observer adjusied $D_{\alpha}$ to be larger. Taking the sign of $\mathrm{W}^{\prime}$ into consideration, the effect of $\mathrm{D}_{\mathrm{I}}^{\prime}$ on $\mathrm{W}^{\prime}$ in Table 1 is that, as predicted from Figure 2, $W^{\prime}$ decreased with increasing $D_{I}^{\prime}$. The effect of $D_{I}^{\prime}$ on $S$, in Table 1, is that, as predicted from Figure 1, S also decreased with increasing $D_{I}^{\prime}$.

The effect of $D_{p}$ on $W^{\prime}, S$, and $D_{\alpha}$, with $D_{I}^{\prime}$ constant, according to the data of Table 1, is that, (1) taking the sign of $W^{\prime}$ into consideration, $W^{\prime}$ increased with increasing $D_{p}$, (2) $S$ also increased with increasing $D_{p}$, and (3) $D_{\alpha}$ did not seem to vary systematically with $D_{p}$. The systematic change in $S$ and $W^{\prime}$ with increasing $D_{p}$ for a constant $D_{1}^{\prime}$ is in agreement with the relations illustrated in Figures 1 and 2. A possible reason for the systematic changes in $W^{\prime}$ and $S$, but not in $D_{\alpha}$, for increasing $D_{p}$
Table 1

Results (in Centimeters) From $\mathrm{D}_{\alpha}, \mathrm{W}^{\prime}$, and $\mathrm{S}$

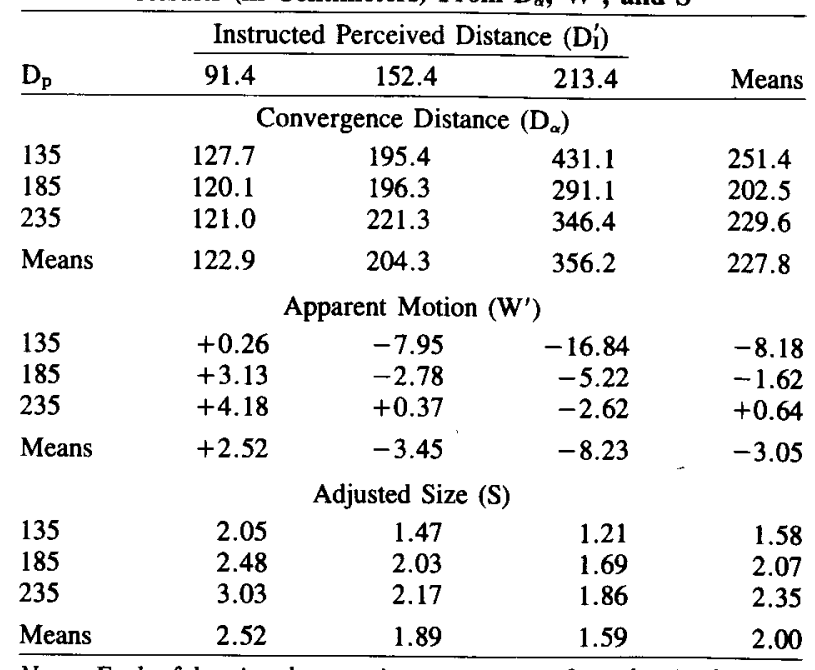

Note - Each of the nine data entries are averages from the six observers in two sessions for each of the nine combinations of $D_{1}^{\prime}$ and $D_{\mathrm{p}}$.

with $\mathrm{D}_{\mathrm{I}}^{\prime}$ constant will be considered in the discussion section.

The conclusions from the above examination of Table 1 are supported by three-way analyses of variance. In an analysis of the effect upon adjusted convergence $\left(D_{\alpha}\right)$ of the factors of sessions, instructed distance $\left(\mathrm{D}_{\mathrm{I}}^{\prime}\right)$, and pivot distance $\left(D_{p}\right)$, it was found that only $D_{I}^{\prime}[F(2,10)=7.01$, $p=.01]$, but not $D_{p}$ or sessions, was significant. In an ANOVA of the effect of the same factors upon apparent concomitant motion $\left(\mathrm{W}^{\prime}\right)$, both $\mathrm{D}_{\mathrm{I}}^{\prime}$ and $\mathrm{D}_{\mathbf{p}}$ were significant $[\mathrm{F}(2,10)=10.79, \mathrm{p}<.01$, and $\mathrm{F}(2,10)=23.77$, $\mathrm{p}<.001$, respectively]. The sessions $\times \mathrm{D}_{\mathrm{p}}$ interaction and the $D_{p} \times D_{1}^{\prime}$ interaction also were significant in this case $[F(2,10)=5.00, p=.03$ and $F(4,20)=3.44, p$ $=.03$, respectively]. Finally, in a similar analysis of the effect upon the size (S) adjusted to appear to be 1 in., $D_{I}^{\prime}$ and $D_{p}$, but not any of the interactions, were significant $[\mathrm{F}(2,10)=8.34, \mathrm{p}=.01$, and $\mathrm{F}(2,10)=11.52$, $\mathrm{p}<.01$, respectively].

The data most relevant to the first objective of this study, that is, a comparison of perceived distances computed from the head motion and size adjustment procedures, are given in Table 2 , in which $\mathrm{D}^{\prime}$ from $\mathrm{W}^{\prime}$ (i.e., $\left.D_{W^{\prime}}^{\prime}\right)$ and $D^{\prime}$ from $S$ (i.e., $D_{s}^{\prime}$ ) are shown as a function of $D_{I}^{\prime}$ and $D_{p}$. Each entry in the body of Table 2 is a value of $D_{w^{\prime}}^{\prime}$ or $D_{s}^{\prime}$ averaged over the two sessions and the six observers. $D_{w^{\prime}}^{\prime}$ or $D_{s}^{\prime}$ always were calculated from each $\mathrm{W}^{\prime}$ or $\mathrm{S}$ obtained in each session from each observer before averaging, that is, values of $D_{w^{\prime}}^{\prime}$ or $D_{s}^{\prime}$, not of $W^{\prime}$ or $S$, were averaged to produce the data of the table. An examination of the means of Table 2 shows a clear, systematic increase in both $D_{w^{\prime}}^{\prime}$ and $D_{s}^{\prime}$ as a consequence of increasing $D_{\mathrm{I}}^{\prime}$. This is consistent with the predictions from Figures 2 and 1, respectively. It also is clear that increases in both $D_{w^{\prime}}^{\prime}$ and $D_{s}^{\prime}$ occurred with increases in $D_{p}$. Although $D_{w^{\prime}}^{\prime}$ is consistently less than $D_{s}^{\prime}$, both of these 
measures of perceived distance increased with increases in either $D_{1}^{\prime}$ or $D_{p}$. Again, these conclusions, from examining Table 2 , are supported by an analysis of variance. A four-factor ANOVA was used to analyze the data upon which Table 2 is based. The dependent variable was perceived distance $\left(D^{\prime}\right)$, whether computed from the task of responding to $W^{\prime}$, that is, $D_{w^{\prime}}^{\prime}$, or from the task of adjusting $S$ to appear equal to $l$ in., that is, $D_{s}^{\prime}$. These two different methods of measuring perceived distance are called the task $(T)$ factor. The significance of the effect upon perceived distance of the factors of sessions, $D_{\mathrm{I}}^{\prime}$, $D_{p}$, and $T$ was analyzed. Only the main effects of $D_{I}^{\prime}$, $\mathrm{D}_{\mathrm{p}}$, and $\mathrm{T}$ were significant $[\mathrm{F}(2,10)=13.12, \mathrm{p}<.01$, $F(2,10)=7.22, p=.01$, and $F(1,5)=8.09, p=.04$, respectively]. The significant effect of $T$ is consistent with $D_{s}^{\prime}$ being larger than $D_{w^{\prime}}^{\prime}$ for the same conditions as noted above in discussing Table 2 . None of the interactions involving $T$ were significant. The significance of $D_{\mathrm{I}}^{\prime}$ and $D_{p}$ as main effects, together with the lack of significance of the interaction of $T$ with either $D_{1}^{\prime}$ or $D_{p}$, indicates that both $D_{w}^{\prime}$ and $D_{s}^{\prime}$ varied as a function of both $D_{\mathrm{I}}^{\prime}$ and $D_{\mathrm{p}}$. In addition, a separate ANOVA was performed on each of the $D_{w^{\prime}}^{\prime}$ and $D_{s}^{\prime}$ portions of Table 2 . The results indicate that $D_{w^{\prime}}^{\prime}$ was a function of both $D_{\mathbf{I}}^{\prime}$ and $D_{p}[F(2,10)=9.44, p=.01$, and $F(2,10)=5.28$, $\mathrm{p}=.03$, respectively]. Also, $\mathrm{D}_{\mathrm{s}}^{\prime}$ was a function of both $\mathrm{D}_{\mathrm{I}}^{\prime}$ and $\mathrm{D}_{\mathrm{p}}[\mathrm{F}(2,10)=10.25, \mathrm{p}<.01$, and $\mathrm{F}(2,10)=$ $3.92, \mathrm{p}=.05$, respectively].

Pearson product-moment correlations between the measures of perceived distance $\left(\mathrm{D}^{\prime}\right)$ obtained in the nine experimental situations using the $\mathrm{W}^{\prime}$ and $\mathrm{S}$ tasks for each observer in each session are shown in Table 3, and the graph of the relation between $D_{s}^{\prime}$ and $D_{w^{\prime}}^{\prime}$ is shown in Figure 5. In Session 2, in which the observers were more practiced than in Session 1, five of the six observers produced correlations that were significant beyond the .01 level $(\mathrm{df}=7)$. The Pearson product-moment correlation between $D_{s}^{\prime}$ and $D_{w^{\prime}}^{\prime}$ was also found for the nine data values shown in Table 2 and plotted in Figure 5. The resulting correlation coefficient was 0.981 .

Table 2

Perceived Distance (in Centimeters) as Calculated From Equation 3 Using the $W^{\prime}$ Data $\left(D_{w^{\prime}}^{\prime}\right)$ and From Equation 1 Using the S Data $\left(D_{s}^{\prime}\right)$

\begin{tabular}{|c|c|c|c|c|}
\hline \multirow[b]{2}{*}{$\mathrm{D}_{\mathrm{p}}$} & \multicolumn{3}{|c|}{ Instructed Perceived Distance ( $\left.D_{1}^{\prime}\right)$} & \multirow[b]{2}{*}{ Means } \\
\hline & 91.4 & 152.4 & 213.4 & \\
\hline \multicolumn{5}{|c|}{ Perceived Distance From $W^{\prime}$ (i.e., $D_{w^{\prime}}^{\prime}$ ) } \\
\hline 135 & 133.4 & 184.9 & 240.8 & 186.4 \\
\hline 185 & 158.1 & 203.1 & 229.9 & 197.0 \\
\hline 235 & 189.3 & 231.0 & 263.6 & 228.0 \\
\hline Means & 160.3 & 206.3 & 244.8 & 203.8 \\
\hline \multicolumn{5}{|c|}{ Perceived Distance From S (i.e., Ds) } \\
\hline 135 & 180.5 & 254.9 & 295.6 & 243.7 \\
\hline 185 & 201.9 & 249.6 & 296.9 & 249.5 \\
\hline 235 & 241.2 & 307.8 & 327.9 & 292.3 \\
\hline Means & 207.9 & 270.8 & 306.8 & 261.8 \\
\hline
\end{tabular}

Note-Each of the nine data entries are averages from the six observers in two sessions for each of the nine combinations of $D_{\mathrm{I}}^{\prime}$ and $D_{\mathrm{p}}$.
Table 3

Pearson Product-Moment Correlations (r) Obtained Between the $D_{\mathrm{s}}^{\prime}$ and $D_{w}^{\prime}$ for Each Observer and Each Session

\begin{tabular}{cllc}
\hline Observer & Session 1 & Session 2 & Session Averages \\
\hline 1 & 0.546 & $0.841^{* *}$ & $0.701^{*}$ \\
2 & $0.793^{*}$ & $0.888^{* *}$ & $0.868^{* *}$ \\
3 & 0.062 & 0.655 & 0.459 \\
4 & 0.655 & $0.856^{* *}$ & $0.754^{*}$ \\
5 & $0.885^{* *}$ & $0.867^{* *}$ & $0.909^{* *}$ \\
6 & $0.866^{* *}$ & $0.905^{* *}$ & $0.910^{* *}$ \\
\hline
\end{tabular}

Note-The Session Averages are the correlations between $D_{s}^{\prime}$ and $D_{w}^{\prime}$ obtained from averaging $D_{\mathrm{s}}^{\prime}$ and $D_{\mathrm{w}}^{\prime}$ over sessions for each observer. The correlation between $D_{\mathrm{s}}^{\prime}$ and $D_{w^{\prime}}^{\prime \prime}$ averaged over both observers and sessions is $0.981 . \quad{ }^{*} p<.05 . \quad *^{*} p<.01$.

\section{Calibration Situations}

The results from the size calibrations were that, on the average, the observers adjusted a vertical separation of $2.42 \mathrm{~cm}(\mathrm{SD}=1.01 \mathrm{~cm})$ to be equal to 1 in. $(2.54 \mathrm{~cm})$. Assuming that physical size in the size calibration was perceived correctly, it can be concluded that, on the average, the observers adjusted the size in the experimental situations to be perceptually $2.42 \mathrm{~cm}$ rather than the $2.54 \mathrm{~cm}$ requested by the experimenter. In other words, the $S^{\prime}$ used in Equation 1 should have been $2.42 \mathrm{~cm}$ rather than $2.54 \mathrm{~cm}$, a value that would have decreased the $D_{\mathrm{s}}^{\prime}$ data of Table 1 by a multiplicative factor of 953 . Thus, the average mean value of $D_{s}^{\prime}(261.8 \mathrm{~cm}$ in Table 2$)$ is changed by this calibration to $249.5 \mathrm{~cm}$.

The average results from the distance calibration were that the observers adjusted a distance of $123.0 \mathrm{~cm}$ (SD $=15.23 \mathrm{~cm})$ to equal $3 \mathrm{ft}(91.4 \mathrm{~cm})$, a distance of $197.4 \mathrm{~cm}(\mathrm{SD}=17.90 \mathrm{~cm})$ to equal $5 \mathrm{ft}(152.4 \mathrm{~cm})$, and a distance of $262.2 \mathrm{~cm}(\mathrm{SD}=30.74 \mathrm{~cm})$ to equal $7 \mathrm{ft}$ $(213.4 \mathrm{~cm})$. Assuming that distance was correctly perceived in the calibration alley, it can be concluded that, on the average, the observers actually adjusted the perceived distance in the experimental situations (by adjusting convergence) to be equal to $4.0,6.5$, and $8.6 \mathrm{ft}$ (an average of $194.2 \mathrm{~cm}$ ) rather than to the instructed perceived distance of 3,5 , and $7 \mathrm{ft}$ (an average value of $152.4 \mathrm{~cm})$.

\section{DISCUSSION}

The data of this study support the validity of the headmotion procedure for measuring apparent distance. The high linear correlation $(r=0.981)$ between perceived distance $\left(D_{w^{\prime}}^{\prime}\right)$, as measured by the $W^{\prime}$ associated with the head-motion procedure, and the perceived distance $D_{s}^{\prime}$, as measured by the adjusted $S$ associated with size-distance invariance, suggests that the two indirect measures of perceived distance vary in a similar manner. The present study provides no explanation, however, as to why $D_{s}^{\prime}$ is consistently larger than $D_{w}^{\prime}$. A possible reason is found in the evidence (Foley, 1968, 1972) that under conditions of binocular observation, $C$ in Equation 1 is larger than unity (rather than being unity, as is assumed in the present computations). If a $C$ of about 1.4 had been used, 


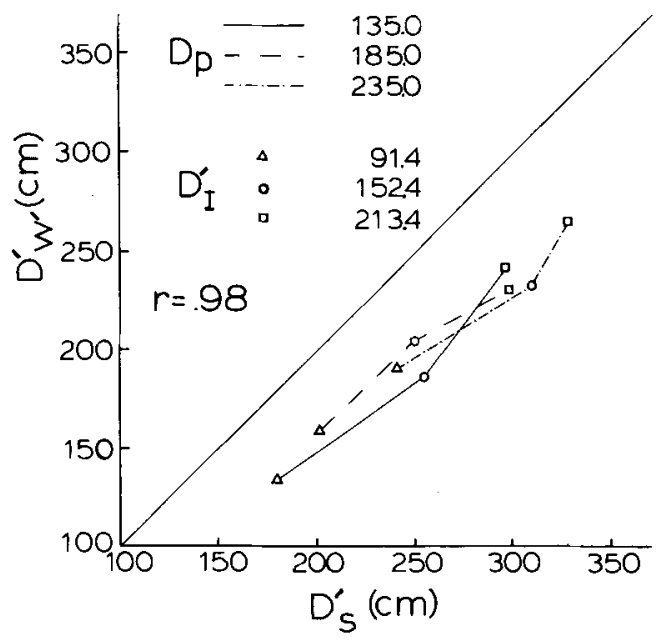

Figure 5. Curves showing the relation between $D_{w}^{\prime}$ and $D_{\mathrm{s}}^{\prime}$ for different values of pivot distance $\left(D_{p}\right)$, with each curve containing the adjustments of the convergence to the same three apparent distances $\left(D_{1}^{\prime}\right)$. The displacement of the data curves from the uninterrupted solid line indicates that the computed values of $D_{s}^{\prime}$ are consisteritly larger than those of $D_{w}^{\prime}$. The fact that the three curves overlap and are continuous with one another, however, strongly suggests that they all are part of the single function relating $D_{5}$ and $D_{w^{\prime}}^{\prime}$. Consistent with Equation 3, the same function relating $D_{w}^{\prime}$ and $D_{s}^{\prime}$ is maintained whether changes in apparent distance are produced by changes in the convergence to the test point or by changes in the depth cues of the test point resulting from changes in the physical distance (D or $D_{p}$ ) of the test point.

the data curves of Figure 5 would have been close to the uninterrupted solid line for which $D_{s}^{\prime}=D_{w^{\prime}}^{\prime}$. It cannot be argued that the high correlation between $D_{s}^{\prime}$ and $D_{w^{\prime}}^{\prime}$ resulted from a similarity in judging the apparent size of a physical separation and the apparent extent of an illusory motion. $D_{s}^{\prime}$ and $D_{w^{\prime}}^{\prime}$ differed as to the origin of the judgments. For the SDIH, perceived size (with a constant sign) changed with perceived distance from the observer's cyclopean eye. The illusory motion (with a variable sign), on the other hand, changed with perceived distance from the pivot distance, a distance which, in the present study, was variable.

The present results extend the findings of a previous study (Gogel, 1979), which examined the relation between these two indirect measures of perceived distance. In that study, in which perceived size was measured by a perceived extent of motion, the fixation was either on the test point or, as a means of varying the perceived distance of the test point, the fixation was on stationary objects at different distances from the test point. This change in fixation in this previous study could have influenced the results. Consistent with the Aubert-Fleischl paradox (Aubert, 1887; Fleischl, 1882), the perceived velocity of the test object, and thus its perceived extent of motion, might have increased when the fixation was not on the moving test object. The present study avoids this problem and extends the comparison between the two indirect measures to the case in which $D_{p}$ as well as $D^{\prime}$ is variable.

The second objective of the present study was to determine whether the apparent-distance/pivot-distance hypothesis, which has been shown to predict the apparent concomitant motion of a single test object in isolation as the head is moved (see Gogel, 1981, 1982), can also predict the apparent concomitant motion of the test object when it is presented in the context of other objects. The issue is not whether the presence of additional objects (in this study the polka dots) contributed to the apparent distance of the test object. It is likely that they did. Instead, the issue is whether the distributions of motions of the images of the polka dots on the eye relative to the test point changed the $\mathrm{W}^{\prime}$ and hence the calculated measure of perceived distance $\mathrm{D}_{w^{\prime}}^{\prime}$, without having actually modified the perceived distance of the test point. As discussed, such a result, if it occurred, might be due to a kind of induced motion produced in the test point as a consequence of its relative retinal motion with respect to the polka dots acting as inducing objects. Depending upon their distances in the visual alley relative to the fixated test point, the retinal images of the different polka dots moved relative to the image of the test point. The number of polka dot images that moved on the retina in a direction opposite to the head motion decreased as the distance of fixation $\left(D_{p}\right)$ increased. It is therefore possible that this factor could have modified the $D_{w^{\prime}}^{\prime}$ measure by modifying $\mathrm{W}^{\prime}$ without actually changing the perceived distance of the test point. If this had occurred, the computation of $D_{w^{\prime}}^{\prime}$, but not of $D_{s}^{\prime}$, would have been affected, the form of the function relating $D_{w^{\prime}}^{\prime}$ and $D_{s}^{\prime}$ would have been different for different values of $D_{p}$, and the $D_{w^{\prime}}^{\prime}$ measure of perceived distance would have been in error. The fact that both $D_{s}^{\prime}$ and $D_{w}^{\prime}$ increased with increasing $D_{p}$ argues against this possibility. Figure 5 permits a more detailed examination of this question.

In Figure 5, the data are plotted as three separate curves with $D_{p}$ a parameter. To a close approximation, each of the three curves is a continuation of the other curves, with the curves overlapping. It will be noted, as indicated by the previous analyses, that $D_{p}$ had a similar effect upon $D_{s}^{\prime}$ and $D_{w^{\prime}}^{\prime}$, with the result that the function relating the $D_{s}^{\prime}$ and $D_{w}^{\prime}$ remained independent of $D_{p}$. Since the only way in which $D_{p}$ could have modified $D_{s}^{\prime}$ was by modifying the perceived distance of the test point, it follows that the effect of $D_{p}$ on $D_{w^{\prime}}^{\prime}$ also is explained by its effect on the perceived distance of the test point. According to the present study, the apparent-distance/pivot-distance hypothesis applies successfully to situations in which many objects in addition to the test object are visible.

In view of the conclusion that $D_{p}$ modified $D_{w^{\prime}}^{\prime}$ only as it changed perceived distance, it is of interest to specify the distance cues that were changed in the present study when the physical (pivot) distance of the point of light was increased. Increasing $D$ (and $D_{p}$ ) of the test point increased the perceived distance expected from absolute motion parallax, absolute accommodation, and absolute brightness. The relative cues that were changed by changing $D_{p}$ were relative motion parallax, relative accommodation, and relative brightness. These latter cues occurred between the test point of light and the polka dots in the visual alley. A normally very effective cue that was not 
directly modified by $D_{p}$, however, was the relative cue of binocular disparity between the test point and the polka dots. It will be recalled from Figure 3 that the convergence to the test point was varied independently of the physical distance $D$ of the display surface. The binocular disparity between the test point and the polka dots, because it was determined by the eye convergence to the test point, was independent of the $D$ (or $D_{p}$ ) of the test point. Thus, whenever $D_{p}$ and $D_{\alpha}$ were different, the distance cue of binocular disparity was in conflict with the cues of absolute and relative brightness, accommodation, and motion parallax as specified by the $D_{p}$ of the test point. It was concluded from the $D_{s}^{\prime}$ and $D_{w^{\prime}}^{\prime}$ results that the latter cues were of some consequence in determining the apparent distance of the test point. It might be supposed, therefore, that an effect of $D_{p}$ would also be evident in the observer's adjustment of the test point to the $\mathrm{D}_{\mathrm{I}}^{\prime}$ values of 3,5 , or $7 \mathrm{ft}$. For example, as $D_{p}$ increased, the $D_{\alpha}$ needed in order to achieve a particular $\mathrm{D}_{1}^{\prime}$ would be expected to decrease. But, according to the results, this did not occur. Although $D_{p}$ changed both $D_{w^{\prime}}^{\prime}$ and $D_{s}^{\prime}$, it did not significantly modify the $D_{\alpha}$ required to achieve a particular $D_{l}^{\prime}$.

How might this effect of $D_{p}$ on $D_{w^{\prime}}^{\prime}$ and $D_{s}^{\prime}$ but not on the $D_{\alpha}$ be understood? A possible answer is found in the attention directed by the observer to different distance cues in the $D_{\mathrm{I}}^{\prime}$ task as contrasted with the tasks involving $\mathrm{W}^{\prime}$ and $S$. For the response to the $D_{\mathrm{I}}^{\prime}$ task, it is likely that the observer decided where the 3,5, or 7-ft distance was in the visual alley and adjusted the convergence until the test point appeared at that distance. In this case, the binocular disparity between the point of light and the polka dots provided a strong cue to perceptually localize the point of light in the visual alley, with less attention paid to the conflicting distance cues provided by the $D_{p}$ of the point. On the other hand, in the task of judging $W^{\prime}$ or of adjusting $S$ to $1 \mathrm{in}$. in apparent height, attention probably was directed to the test point or points with less concern regarding their perceived distances with respect to the alley. As a consequence, for these latter tasks, binocular disparity would be less effective, and the distance cues associated with $D_{p}$ would have a greater role in determining the response. This explanation invokes the role of attention in weighting the contribution of distance cues. Consistent with this analysis, a number of other studies have shown that attention can modify the weight that an observer gives to a particular source of visual information (Brussell, 1973; Brussell \& Festinger, 1973; Coren \& Porac, 1983; Festinger, Coren, \& Rivers, 1970; Gogel, 1965, 1967; Gogel \& MacCracken, 1979; Gogel \& Tietz, 1976; Tsal, 1984). If supported by additional research, this effect of attention on the weighting of distance cues may prove to be of considerable importance in understanding the effect of task variables and instructions on perceived distance.

According to the apparent-distance/pivot-distance hypothesis, the apparent motion between two objects in the visual field cannot be specified by their retinal motion alone. Indeed, the illusory motion between two ob- jects concomitant with a motion of the head can be in a direction opposite to that expected exclusively from their relative motions on the retina. This conclusion, directly demonstrated in a previous study (Gogel, 1980), follows inferentially from the present results. It can be shown geometrically that the relative motion on the retina of images of physically stationary objects, for example, a test object and a particular polka dot in the present study, is determined entirely by the physical positions of the objets relative to the observer. As illustrated unambiguously in the present study, the apparent motion of the test point, and thus its apparent motion relative to the particular polka dot, can be changed in both magnitude and direction by changing the apparent distance of the test point (with $\mathrm{D}$ or $D_{p}$ constant). Thus, the test point under some conditions must have appeared to move in one direction relative to some of the polka dots, while the apparent relative motion expected solely from the relative retinal motion between the test point and these polka dots was in the opposite direction. This phenomenon can be explained more formally. In the the context of lateral motion of the head, the relative retinal motions of two physically stationary objects (e and $f$ ) located at a near $D_{e}$ and farther $D_{f}$ distance can be shown to be equal to the difference between their pivot angles $\phi_{\mathrm{e}}$ and $\phi_{\mathrm{f}}$, where approximately, in radians,

$$
\phi_{\mathrm{e}}-\phi_{\mathrm{f}}=\mathrm{K} / \mathrm{D}_{\mathrm{e}}-\mathrm{K} / \mathrm{D}_{\mathrm{f}}=\mathrm{K}\left(1 / \mathrm{D}_{\mathrm{e}}-1 / \mathrm{D}_{\mathrm{f}}\right) .
$$

Also, from Equation 2, the apparent relative motion between objects $e$ and $f$ is

$$
W_{e}^{\prime}-W_{f}^{\prime}=K\left(D_{f}^{\prime} / D_{f}-D_{e}^{\prime} / D_{e}\right)
$$

Several predictions follow from Equations 4 and 5. If $D_{e}^{\prime}$ $=\mathrm{D}_{\mathrm{f}}^{\prime}$, then

$$
W_{e}^{\prime}-W_{f}^{\prime}=K D^{\prime}\left(1 / D_{f}-1 / D_{e}\right) .
$$

A comparison of Equations 4 and 6 shows that the retinal and apparent relative motions are proportional if, and only if, the objects are perceived to be at the same distance $\left(D^{\prime}\right)$, regardless of whether or not they are physically equidistant. A perception of motion proportional to the retinal motion is a consequence of perceiving the objects as equidistant. The perception of equidistance, in this case, is essential to the proportionality. Another prediction is that the perceived relative motion can be in the same direction as, or opposite to, that predicted from the retinal motion depending upon the relation between $D_{f}^{\prime} / D_{f}$ and $D_{e}^{\prime} / D_{e}$ in Equation $5 .{ }^{4}$ For example, if $D_{f}^{\prime}>D_{f}$ and $D_{e}^{\prime}<D_{e}$, the direction of the apparent relative motion actually obtained will be opposite to that predicted from the relative retinal motion (see Gogel, 1980). The validation of Equation 2 as indicated by the present study and its expansion into Equation 5 strongly asserts the inability to equate perception with the retinal motion alone. To predict the magnitude and direction of apparent concomitant motion, in- 
formation as to apparent distance of the test points must be available. It will be noted that Equations 1, 2, 5, and 6 involve the relation between different perceptions, that is, perceptions of distance and motion. Such perceptual equations, as distinguished from psychophysical equations, with the latter limited to the relation between physical and perceptual variables, seem to be essential if the relation between proximal stimuli and the perceptual response is to be understood.

\section{REFERENCES}

Aubert, H. (1887). Die Bewegungsempfindungen. Pflügers Archiv für gesamte Physiologie, 40, 459-480.

BRUSSELl, E. M. (1973). Attention, brightness contrast, and assimilation: The influence of relative area. Perception \& Psychophysics, 14, 325-333.

Brussell, E. M., \& Festinger, L. (1973). The Gelb effect: Brightness contrast plus attention. American Journal of Psychology, 86, 225-235.

Coren, S., \& Porac, C. (1983). The creation and reversal of the MüllerLyer illusion through attentional manipulation. Perception, 12, $49-54$.

DUNCKer, L. (1929). Über induzierte Bewegung. Psychologische Forschung, 12, 180-259. [Translated and condensed in W. Ellis (1950). Source book of Gestalt Psychology (Atlantic Highlands, NJ: Humanities Press) Selection 12.]

Festinger, L., Coren, S., \& Rivers, G. (1970). The effect of attention on brightness contrast and assimilation. American Journal of Psychology, 83, 189-207.

FLEISCHL, E. (1882). Physiologisch-optische Notizen. Sitzungsberichte der Akademie der Wissenschaften, 89, 17-25.

FoLEY, J. M. (1968). Depth, size and distance in stereoscopic vision. Perception \& Psychophysics, 3, 265-274.

FolEY, J. M. (1972). The size-distance relation and intrinsic geometry of visual space: Implications for processing. Vision Research, 12, 323-332.

GiLINSKY, A. S. (1951). Perceived size and distance in visual space. Psychological Review, 58, 460-480.

GoGEL, W. C. (1965). Size cues and the adjacency principle. Journal of Experimental Psychology, 3, 289-293.

Gogel, W. C. (1967). Cue enhancement as a function of task set. Perception \& Psychophysics, 2 , 455-458.

GoGEL, W. C. (1976). An indirect method of measuring perceived distance from familiar size. Perception \& Psychophysics, 20, 419-429.

GOGEL, W. C. (1979). The common occurrence of errors of perceived distance. Perception \& Psychophysics, 29, 2-11.

GoGEL, W. C. (1980). The sensing of retinal motion. Perception \& Psychophysics, 28, 155-163.

GOGEL, W. C. (1981). The role of suggested size in distance responses. Perception \& Psychophysics, 30, 149-155.

GOGEL, W. C. (1982). Analysis of the perception of motion concomitant with a lateral motion of the head. Perception \& Psychophysics, 32, 241-250.

Gogel, W. C., \& MacCracken, P. J. (1979). Depth adjacency and induced motion. Perceptual and Motor Skills, 48, 343-350.

GogeL, W. C., \& TIETZ, J. D. (1976). Adjacency and attention as determiners of perceived motion. Vision Research, 16, 839-845.

GoGEL, W. C., \& TIETZ, J. D. (1979). A comparison of oculomotor and motion parallax cues of egocentric distance. Vision Research, 19, 1161-1170.

HAY, J. (1971). Does head-movement feedback calibrate the perceived direction of optical motions? Perception \& Psychophysics, 13, 284-292.

KilPatrick, F. P., \& ItTElson, W. H. (1953). The size-distance invariance hypothesis. Psychological Review, 60, 223-231.

MacCracken, P. J., Gogel, W. C., \& Bum, G. S. (1980). Effects of posthypnotic suggestion on perceived egocentric distance. Perception, 9, 561-568.
TIETZ, J. D., \& GoGEL, W. C. (1978). Adaptation to apparent concomitant motion in the absence of physical or retinal motion. Bulletin of the Psychonomic Society, 12, 1-4.

TsaL, Y. (1984). A Mueller-Lyer illusion induced by selective attention. Quarterly Journal of Experimental Psychology, 36A, 319-333.

Wallach, H., \& Kravitz, J. (1965). The measurement of the constancy of visual direction and of its adaptation. Psychonomic Science, 2, 217-218.

Wallach, H., Yablick, G., \& Smith, A. (1972). Target distance and adaptation in distance perception in the constancy of visual direction. Perception \& Psychophysics, 12, 139-145.

\section{NOTES}

1. In previous articles (e.g., Gogel, 1982), the term $m^{\prime}$ rather than $\mathrm{W}^{\prime}$ was used to indicate the magnitude of the apparent concomitant motion of the test point. In the present article, $W^{\prime}$ is used rather than $m^{\prime}$ to avoid confusion between this term and the symbol $\mathrm{m}$ used in Figure 2 to indicate a middle-distance.

2. It is possible to change $D_{p}$ while keeping $D$ constant by physically moving the test object concomitant with the motion of the head (see Gogel, 1976,1982 ). In the present study, however, $D_{p}$ was modified by changing $\mathrm{D}$ with the test point of light always physically stationary.

3. It is reasonable to assume that there was no consistent error in the measurement of $\mathrm{W}^{\prime}$ using the tactual procedure in which the separation of the two rods was used by the observer to indicate the magnitude of the apparent lateral motion of the point. This assumption is justified by a study in which the results obtained by this procedure were found to be very similar to those obtained by visually duplicating the apparent concomitant motion by a physical lateral motion adjusted while holding the head stationary (Gogel \& Tietz, 1979). In that study, D' calculated from $\mathrm{W}^{\prime}$, as obtained by the adjusted separation of two posts by touch or by a duplication procedure, averaged over the six observers and three distances of a test point were 70.7 and $71.7 \mathrm{~cm}$, respectively.

4. Whenever the sign of the relative retinal motion $\left(\phi_{\mathrm{e}}-\phi_{\mathrm{f}}\right)$ in Equation 4 is the same as the relative apparent motion $\left(W_{e}^{\prime}-W_{f}^{\prime}\right)$ in Equation 5 , the relative apparent motion predicted from $\phi_{e}-\phi_{\mathrm{f}}$ is opposite to that expected from Equation 5. The reason for this is that the direction of distal motion is inverted in its retinal representation.

\section{APPENDIX}

A Glossary of Terms

Term Definition

D Physical distance of the test object from the observer

S Physical size of the test object

K Lateral extent of the physical motion of the observer's head

$D_{\alpha} \quad$ Physical distance at which the optical axes of the two eyes converge (called the convergence distance)

$D_{p} \quad$ Physical distance around which the direction of the test object from the observer pivots as the head is moved laterally (called the pivot distance)

$\phi_{\mathrm{p}} \quad$ Angle formed at the pivot distance by the physical directions from the observer to the test object at the extremes of head motion

D' Perceived distance of the test object from the observer

$S^{\prime} \quad$ Perceived size of the test object

$W^{\prime} \quad$ Perceived lateral motion of the test object concomitant with the physical lateral motion of the head

Di Perceived distance of the test object from the observer which the observer is instructed by the experimenter to achieve by varying the convergence to the test object 
$D_{w}^{\prime} \quad$ Perceived distance of the test object from the observer, as calculated from the apparent-distance/pivot-distance hypothesis, using the observer's perception of the motion $\left(W^{\prime}\right)$ of the test object

$\mathrm{D}_{\mathrm{s}}^{\prime} \quad$ Perceived distance of the test object from the observer, as calculated from the SDIH, using the observer's ad- justment of the physical size (S) of the test object to appear 1 in. in extent

Note-Subscripts $n, m$, and $f$, when used in the text or figures with $S, D, S^{\prime}$, and $D^{\prime}$, indicate a physical or perceived size or distance associated with the test object at a near, middle, or far distance, respectively, from the observer.

(Manuscript received August 6, 1984;

revision accepted for publication December 3, 1984.) 